\title{
Drunk on risk: how the chief medical officers' alcohol guidelines are demonising drink
}

In this letter (BMJ 2016;352:i1175, doi:10.1136/bmj.i1175) the entire second paragraph except for the first sentence should be in quotation marks and attributed to Sally Davies. This omission of quotation marks, which should begin at "If you take 1000 women ..." and end at "it's hard science," is the result of an editorial error. 